\title{
Chapter 3 \\ Designing Edukata, a Participatory Design Model for Creating Learning Activities
}

\author{
Tarmo Toikkanen, Anna Keune, and Teemu Leinonen
}

\begin{abstract}
Closing gaps between visionary ideas and classroom practice was the key achievement of the design research and work of the iTEC project. The design activities were based on the traditions of Scandinavian participatory design, activity theory, service design, artistry, and a specific view on learning design. Within iTEC, the design research and work brought forward the concept of Learning Activities as a useful mode of communicating new ideas to teachers that provided both challenges and support for overcoming those challenges. Evaluation results showed that Learning Activities were extremely successful. This success led to the need to ensure the continuation of Learning Activity design and production beyond the project. The design approach for creating the Learning Activities was captured for educators in the Edukata toolkit. Radical simplification yielded a model that seems to be valuable for teachers even with small amounts of training. However, the full impact of this model and its applicability in the diverse school learning settings across Europe remains to be validated. In this article we present the design research process and one of its main results: the Edukata toolkit for teachers to design their own Learning Activities to bridge the gap between tie visionary ideas and classroom practice.
\end{abstract}

Keywords Participatory design $\bullet$ Design $\bullet$ Learning activity $\bullet$ Prototyping $\bullet$ Change management $\bullet$ Teaching $\bullet$ Learning

\section{Introduction: Design and Pedagogical Research}

Curricular requirements in European classrooms are handed to teachers top down, although educators are often invited to take part in the process of defining them. The top down model is an obvious hindrance to teacher-led innovation. From our experiences in Finland, where teachers have much autonomy on classroom activities, we see teacher-led innovation as a crucial part of developing school practices and culture. Teachers often know their students and their needs, understand the subject matter and can make well-informed calls about how to design their classroom activities.

T. Toikkanen $(\varangle) \bullet$ A. Keune $\bullet$ T. Leinonen

Aalto University, Espoo, Finland

e-mail: tarmo.toikkanen@aalto.fi; anna.keune@aalto.fi; teemu.leinonen@aalto.fi 
In the iTEC project Lewin and McNicol (2014) have found empirical evidence that well designed Learning Activities following a certain template are valuable tools for teachers to challenge their own established practices and to try out new methods and tools. The results demonstrate that the experiments by teachers offer significant improvements in the students' working culture, engagement, motivation, and ultimately, learning outcomes. Evaluations from over 2500 classroom pilots have indicated that the Learning Activities designed in the iTEC project are effective at enthusing teachers and students, affecting change in classroom practices, and prompting other teachers to adopt similar practices. They also encouraged teachers and students to start using novel ICT tools and services, and to use the tools in the way the educational designer intended for them to be used so that they benefit the learning process. Furthermore, when teachers are supported to design their own Learning Activities with proper facilitation and guidance, results are even better (Lewin and McNicol 2014).

Our design research question was:

\section{What kind of support, training, materials, and experience is needed for teach- ers to create their own Learning Activities that integrate visionary ideas into classroom teaching and learning?}

Our hypothesis is Edukata, a set of guidelines targeted towards teachers, which are intended to enable them to better design and reformulate their teaching practices in collaboration with students and other expert educators. Edukata is based on the group's design-research approach called "Research-based design with prototypes", described in Leinonen et al. (2008).

This design-research approach has been used and developed by the research group since 1997 and is continually being developed (see Fig. 3.1). The group is multi-disciplinary, consisting of designers, educators, engineers, psychologists and cognitive scientists. The method has been used to design and implement software prototypes for reflection, knowledge building, and Open Educational Resource (OER) authoring, as well as physical environments, future scenarios,

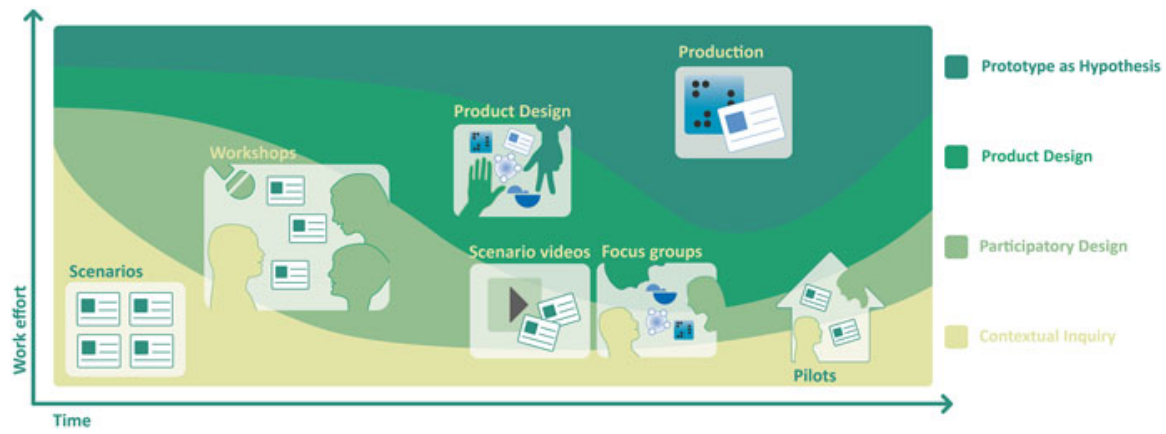

Fig. 3.1 An overview of the research-based design methodology and the design methods, as adapted for the iTEC project and called "Research-based design with prototypes". All four modes of work proceed in parallel, with the focus of work shifting between them as time passes. Concrete design and research activities from cycle 1 are overlaid 
and educational practices (see e.g., Leinonen et al. 2003; Ford and Leinonen 2009; Keune and Leinonen 2013; Durall and Toikkanen 2013). The approach is constructed of, and builds on, four design approaches: tool design, educational design, participatory design and learning design.

Our philosophy on tool design draws on Engeström's (1987, 1999) emphasis that a tool may provide subjects with new abilities to act with objects around them, as well as being part of the larger socio-cultural context that is conditioned with various constraints. Similarly to the idea of a tertiary artefact, which can impact the way in which a person may see, interact with, and shape the world (e.g., Cole 1996), in the best case, this means that the tools created by a designer affect the sociocultural system within which the tool is situated, and are affected and modified by the same (Leinonen 2010).

Our philosophy on design in and for education draws from Rittel's (1972) view that each challenge can have multiple solutions, and that attempts to solve challenges often construct new, potentially even more complex challenges. To differentiate from problem-based approaches, we value the idea of the designer as someone who creates desired additions to the present state, as opposed to merely reactively solving problems as they emerge. We acknowledge Schön's (1987) view on artistry, meaning the way designers combine their domain understanding and design expertise with intuition, often leading to surprising results, which might not be logically tracked back to the starting point. We also agree with Nelson and Stolterman (2003) in that the designer's actions are intentional contributions to the situation and the designer is an active participant in the change process. Nelson and Stolterman (2003) schematize the designer's intentions in relation to (1) helping (fixing, assisting, patronizing), (2) art (persuading, influencing, manipulating, proselytizing), (3) science (describing, explaining, predicting, controlling), and (4) service (serving, conspiring, emphatizing). Of these four designer intentions, our group's methodological approach focuses strongly on service intentions (Leinonen 2010).

Our way of utilizing participatory design is based on the Scandinavian approach to systems design, which considers it important for those stakeholders who might be affected by the new tools to genuinely participate in the design. Following Ehn and Kyng (1987), we see the people for, and with whom, design is practiced and created as primary drivers for realistic and working innovation. For this to work, the designer needs to spend time with the people in question and learn about their everyday life situations, in place of doing laboratory experiments (Leinonen 2010). We fully acknowledge that design challenges and their solutions are highly context-specific (Muller and Kuhn 1993).

In terms of learning (and teaching) design, we see that it is challenging to present and build on the complexity and messiness of teaching and learning (see e.g., Conole 2010). The attempt to downplay this messiness often leads to schematic representations of teaching, rather than empowering teachers to design their work practices. Our research method, and indeed Edukata, steer away from connections to such patterns, leaving many details open for teachers to complete as they see best. We acknowledge that this makes exact comparison and benchmarking rather difficult with the huge variety of approaches and results, but feel this space for innovation is critical. 


\section{Designing Learning Activities for Piloting}

Edukata has been developed as part of the Innovative Technologies for an Engaging Classroom project (iTEC), a 4-year pan-European project. The workflow of iTEC was planned to be a straightforward 5-phase iterative process, in which each phase was intended to include four parts: (1) create Learning Scenarios, (2) design software and learning design prototypes based on the scenarios, (3) pre-pilot and pilot the prototypes in classrooms across Europe, and (4) evaluate the pilots. As the plans were implemented they needed to be adapted and changed.

The five piloting cycles gave project partners opportunities to learn from past cycles and to better serve the overall aim of the project. The first cycle was smaller than the later ones, both in scale of piloting activities and scope of challenges. Specifically, organizational challenges (such as combining two classes into a single course) were excluded from the first cycle, so teachers would not have to face challenges that they alone cannot overcome. The following cycles were each larger in scale. Significantly more schools, teachers and classrooms were involved, and the level of technical and pedagogical challenges increased, this time including any and all challenges that were raised in the design work.

\section{The Design Process}

During each of the five piloting cycles, the following design activities took place. A more detailed description can be found in the project's deliverable D3.1 (Keune et al. 2011).

- Each cycle's design work began with scenario analysis using the wall method (see Fig. 3.2). All scenarios were printed and placed on a wall. The team spent several sessions going through the scenarios, highlighting interesting passages, noting similarities, and comparing the scenarios to the state-of-the-art.

- Distributed participatory design workshops took place in most piloting countries. The scenarios were divided among the pilot countries so each had 2-3 scenarios to analyse. The national coordinators translated the scenarios and presented them to a group of teachers, following the guidelines developed by our team. The facilitators were encouraged to adapt the guidelines to their particular facilitation context, for example in relation to the location for facilitating the workshops (e.g., in schools or in ministry facilities). The ensuing conversations were recorded, and the coordinators wrote English summaries of the conversations, which they sent back to us. These summaries allowed us to understand differences in teaching practice and culture in various countries, and to see which aspects of the scenarios were appealing and which challenges teachers foresaw.

- The English summaries were added to the wall. We received 2-4 summaries for each scenario. Another round of analysis ensued, where we had to make hard design decisions on what seemed to be important; which challenges we should 


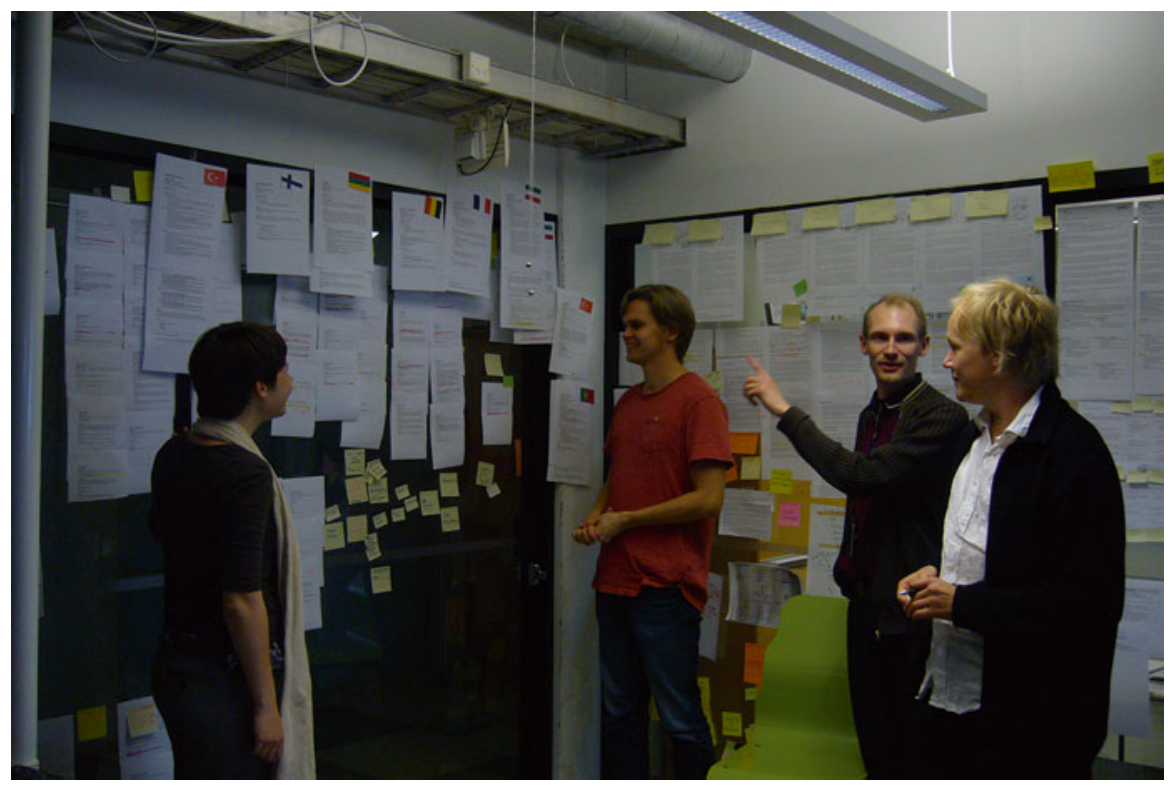

Fig. 3.2 A central but low-tech mode of work is the wall method, where all pertinent information is placed on walls, so they are constantly visible. During design sessions, even a large group can see everything, it is easy to point to individual items, and notes can be added using sticky notes, pens, and highlighters

try to address or circumvent; and what kinds of support teachers would eventually receive from the project were they to try to implement essential segments of these scenarios.

- Prototyping work followed, where both technical prototypes (i.e., software tools with partially functional interfaces) and teacher guidelines for using the software prototypes in relation to the scenarios were designed and developed.

- Focus groups and interviews with teachers and headmasters were held periodically to gauge both the level of innovation and amount of support being built to the prototypes. Focus groups were formed openly from the network of teachers involved in the project. Invitations were sent via national coordinators, social media channels, partner community sites, and so on. Some focus groups were organized partly online and offline to allow more people to join and share experiences.

- Pre-pilots were organized in most piloting countries, in which one or two teachers from each country participated. These were teachers who were confident users of Information Communication Technology (ICT) and had advanced pedagogical skills, so they could work with rough prototypes (i.e., software that is not fully developed and may have parts that do not function fluently at all times), and report back to us any problems they experienced. 
- For us, each project cycle, which marked an iteration, ended with product design, where we took all the feedback we had; decided (as a project consortium), which pre-piloted prototypes should be scaled up to full scale pilots; and polished those prototypes sufficiently to allow average teachers to make use of them.

This general process was followed in all piloting cycles. The details and guidelines evolved as we gained more experience with working with the national coordinators, who were responsible for managing the project in each piloting country, and with the teachers who participated in pre-pilots and pilots. The guidelines and practices were also shaped by the feedback of the teachers and national coordinators. In later cycles we started doing participatory design workshops with pupils, organized online focus groups, and varied the process to maximise its usefulness.

\section{An Example of a Surprising Design Outcome: TeamUp}

The design process outlined earlier takes a great deal of resources and time. To illustrate the concrete benefits of such an involved process, we will describe just one design outcome from the very first piloting cycle.

Twenty mini-scenarios were developed by iTEC partners across Europe during a scenario development workshop, organised by the iTEC partner organisation Futurelab (see Chap. 2). Of the 20 mini-scenarios, the nine most convincing and desirable ones were identified using a prioritisation protocol devised by iTEC partners. These were then fleshed out by Futurelab into detailed scenarios. These detailed scenarios presented the basis of the first cycle design process.

Six of the nine scenarios described the pupils working in small teams. Teamwork was taken for granted, and just mentioned in passing, as can be seen in the example scenario in Fig. 3.3. None of the expert pedagogues nor our design team, who participated in the scenario development workshops considered that this might be a challenge.

When analysing the participatory design workshop summaries from various countries, it became obvious that in most European countries, having pupils work in small teams was not a common practice, and was seen as a real challenge. Teachers from several countries pointed out that they normally do not facilitate teamwork exercises; that following all of the teams and guiding them is a lot of extra work for which the teachers do not have time; and that teams are often dysfunctional, with free-riders or friendship cliques making productive teamwork difficult.

This surprising finding lead us to reconsider the content for the first piloting cycle. Pedagogical experts agreed that teamwork is a useful form of learning without recognizing the challenges it may pose in practice, whereas teachers, by and large, saw teamwork as a foreign, time-consuming and problematic mode of working. No teacher denied the benefits of teamwork, but the practical challenges they saw were a clear showstopper. 
"Ms Rossi, a science teacher, has been liaising with the geography teacher and they have noticed that their students need to develop a more in depth understanding of the local natural environment and wildlife. Ms Rossi has also noticed that although her class works well as individuals, they would benefit from more group learning. She decides to get the group to work collaboratively on a problem-based activity to do with nature and the local environment. When deciding on a specific activity for the class she liaises with the geography teacher to ensure the chosen activity could also support learning in geography. She sets her class the challenge of finding out why the population of ladybirds has decreased in the school grounds over the last year.

Carmen, a student, goes outside with her group to collect real data to help the class's investigation. Each group member has a different role and a different instrument to capture authentic data. Carmen uses her mobile phone to capture images of the areas where most ladybirds live, whilst others in the group record the temperature and survey habitats. Ms Rossi lets the students work together in groups so she can take the role of observer and coach. This helps her understand what skills the students need to practise. She notes down what skills the students need to develop to help her design future learning activities. She realises the group need more training on using instruments without disturbing wildlife, and also how to set specific group goals.

After gathering a series of photos Carmen comes back to class with her group and they share their data and findings with each other. They get some specific support from Ms Rossi on how to use a software package to draw conclusions from the group's numerical data. Having drawn their conclusions, the group choose to create a short film from their photos and data to share their findings with other students in the class. They work together using laptops and a web tool to create a short digital film explaining what they found. Carmen and another student upload their photos while the rest of the group write a script to present their findings. They each record a part of the presentation script and use the automatic editing software on the web tool to create the film. This film is posted on the school's learning platform for the class to view for homework, and also for students in a geography class, who are doing similar work, to comment on. The group also decide to post it on the public area of the learning platform so they can show their parents/carers when they get home."

Fig. 3.3 Example scenario narrative from the first cycle of iTEC, titled "Outdoor study project", written by iTEC partner organization FutureLab in the UK

We had to convene our entire research group to ponder this situation. Finally, we decided that making teamwork a key feature of cycle one's piloting, and providing explicit support for forming teams and for following the teams' study progress would be of most value to the piloting work and for the project.

We drew inspiration from the collaborative progressive inquiry framework (Hakkarainen 2003) and included its ideas on maximizing student motivation by forming interest-based teams to work on specific topics. We also tried to tackle teachers' objections over the time they need to spend following each team's progress with their study projects. The concept became a technological prototype, and finally a fully functional product called TeamUp (see Fig. 3.4). 


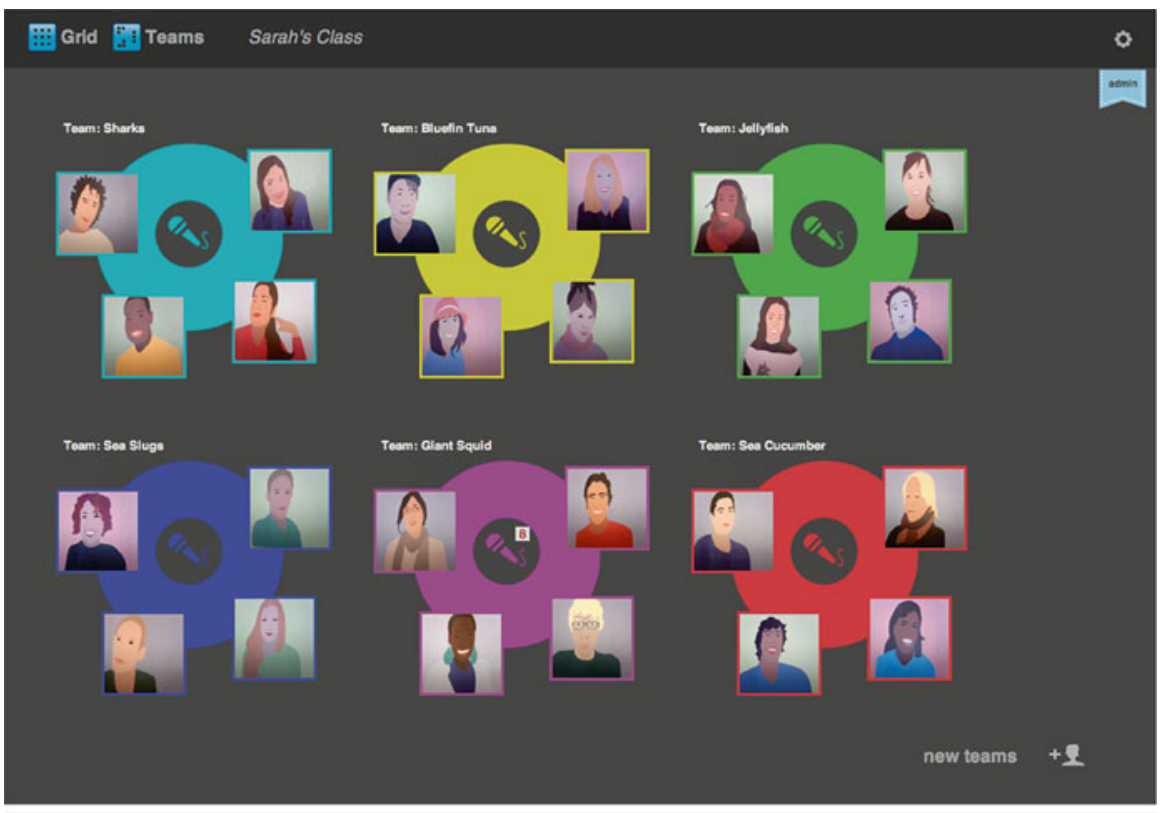

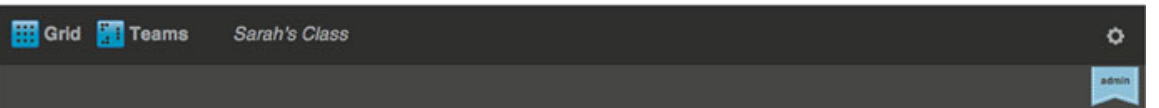

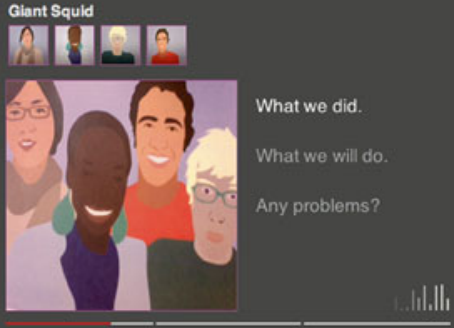

$0,00 / 000$

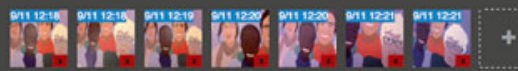

Fig. 3.4 Screen shots from the TeamUp tool with fictional, drawn characters. In real use, photos of students would be used. On the top is the team view, where the teacher and the students can see the team compositions. On the bottom is the view of a single team, with the controls to create new status updates, and to listen to existing ones 
TeamUp is a web-based application that uses a complex algorithm to form heterogeneous, interest-based teams. Additionally, the application includes a feature for teams to record and share audio-visual updates of their work, the challenges they encountered, how these may have been overcome, and what they are planning on doing next (which follows agile stand-up meeting practices). The recordings can be no longer than $60 \mathrm{~s}$. This time limit was intentional. We intended to support students to focus their summaries of their work, and aimed to ensure that a teacher with a class of, say, seven teams needs to spend no more than 7 min between lessons to get an update on the teams' progress. TeamUp also became the first tool to support student reflection, which became a major trend in the following iTEC pilot cycles. TeamUp is further described in both Keune et al. (2011) and Leinonen et al. (2014).

Although the design of TeamUp was intended to address challenges related to forming and following learning teams' progress, during the piloting we noticed that forming teams was not a universal challenge for all teachers. Especially experienced teachers mentioned that they are able to form functional teams without the support provided by TeamUp. However, the possibility to follow the teams' progress and the possibility to surface students' voices for reflection was highlighted as empowering by teachers and students. Therefore, in further developing TeamUp, the feature for forming teams was backgrounded and the feature for sharing team recordings was foregrounded in the interface. These changes made to the tool are examples of the research-based design approach's flexibility and on how the prototypes and tools created in the research work are partly communicating the research results.

\section{Creating the Concept of Learning Activities}

We faced our first challenge with the research in the spring of 2011, during the first cycle. While the scenarios were inspiring and challenging, and had started the creation of several technical prototype ideas (such as TeamUp, ReFlex, Ambire, Plates; see more details in Leinonen et al. (2014), we faced a problem not foreseen during the project-planning phase. What exactly would the piloting teachers be provided with so that they would be challenged as well as supported during their pilots?

With the diverse challenges mentioned by teachers in relation to the scenarios including questions on how to implement them (see D3.1: Keune et al. 2011), the scenarios on their own did not seem sufficiently supporting. The scenarios highlighted visionary ideas in a general narrative structure without mentioning many practical details or challenges that would support teachers in their attempts to implement the visionary ideas. The scenario in Fig. 3.3 is an example of a good quality scenario, conveying the idea of learning and teaching science content outside through narrative devices. However, the scenario skims over the details and practical advice, generalizing the context specific nature of teaching and learning across diverse European settings. Participatory design work with teachers highlighted many issues and challenges teachers saw with implementing the scenarios, some of 
which were even surprises to the experts who had created the scenarios (see previous section for an example).

The first idea was to rewrite the scenarios into Learning Stories, which would be more concrete, contain tips, notices, good practices, scheduling information, options for various tools and technologies, and so on. Drafting the first cycle's Learning Stories revealed that they would be too unwieldy. As each scenario contained many challenging elements, rewriting all of them in more detail would create very long stories, with lots of details obfuscating the visionary ideas. Moreover, as the scenarios shared elements (for example, most scenarios had students working in small teams), each story would end up containing many of the same details.

A workable solution emerged when the details were separated into modules. Each story was constructed with a story arc to present a narrative approach to the ideas, and to exemplify an implementation of particular ideas. All the details for various challenging elements were packed into separate modules, which the stories shared (examples: Reflection, Design brief, Ad-hoc collaboration, Working with outside experts). The term Learning Activity was deemed a good title for the modules, as existing uses of that term did not tie it to divergent preconceived notions, and teachers' intuitive understanding of the term was close enough to its use in this context.

When writing the first cycle's Learning Activities, the design team was very conscious of the tone and method of addressing teachers. While we as designers might have a broad view of the changing educational sector and may have good ideas for teachers to try out, we were aware that it is the teachers who are the experts of their profession and practice, their students, and know what may or may not work. Instead of telling teachers what to do in the pilots and pre-pilots, we decided to rely on their expertise as designers of their own teaching and learning, and merely provide them with new ideas, support, reassurance, and advice, packaged into the Learning Activities. In working with the Learning Activities, e.g., how to interpret them in practice, we gave them the freedom to choose which ones to try and how without strict limitations. This approach made detailed analysis of pilot activities challenging, but was essential in unleashing the creative potential of the teachers, empowering them to decide what to do, and in turn pass that empowerment on to their students.

Another aspect of Learning Activities had to do with their wide audience. We intended the Learning Activities to be used in classroom pilots in 12-16 European countries by teachers with very different didactic methods, technology experiences, and pedagogical approaches. Each Learning Activity had to be written in a way that it would be challenging for experts without fending off beginners by being too challenging. Any single activity, for example students keeping a learning diary in blogs, may be routine for some teachers, and completely new to others. The Learning Activity presenting this concept needed to provide an entry-point for the novices, as well as additional depth and challenges for those already experienced with similar activities. The example in Fig. 3.5 contains many aspects of a Learning Activity that a teacher may choose to include in their own teaching. A teacher not familiar with teamwork might simply split the students into teams and follow their progress, while a more experienced teacher would use the more advanced suggestions in creating motivation-optimized heterogeneous teams. 
You divide the class into small teams of 4-5 learners that are optimal for collaboration. Each team has their own topic of inquiry that is related to the theme of the course. You let the learners suggest topics they are interested in and use the TeamUp tool to match learners and topics, using information stored in mental notes.

- Preparation

Set up the TeamUp tool for your class by adding names, portraits and mental notes of learners. See TeamUp tool manual, part 1 'Add and edit learners' for more information.

Your learners will be working in teams of 4 , each team with a specific topic. Plan your course (or part of it) accordingly.

Decide whether you grade teams or individuals.

- Introduction

Present the theme of the course in a way that gives students some basic information, but leaves open many questions.

Ask learners to think about what they would like to study in this theme.

- Activity

Team work usually spans multiple lessons, often an entire course. Ask students to suggest topics for inquiry. Use your judgment to rephrase, alter or reject suggestions.

Collect topics in the TeamUp tool.

Let learners vote for their favourite topic and create the teams. See TeamUp tool manual, part 2 'Forming teams'.

Ask learners to start their teamwork.

Starting each lesson, show the TeamUp team view to remind everyone of the teams and their topics.

- Assessment

Include contributions to teamwork into your assessment.

You may brainstorm assessment criteria with the learners.

Fig. 3.5 Example Learning Activity narrative from iTEC cycle 1, called "Teamwork"

Title
Summary
Learning outcomes
Motivation: teacher, student
Reasons for using technology
Guidelines (including required time, preparation, assessment)
Technology support
Technical details

Fig. 3.6 Learning Activity template for cycle 1. Items in italics changed as work progressed through the cycles

The Learning Activity in Fig. 3.5 includes a short introductory paragraph, presenting the general frame of the activity, a section for preparing the activity before class, and one for introducing the activity to the class, the main activity description, and, finally, ideas for assessment. Figure 3.6 presents additional aspects that were included in the descriptions of Learning Activities, such as potential learning 


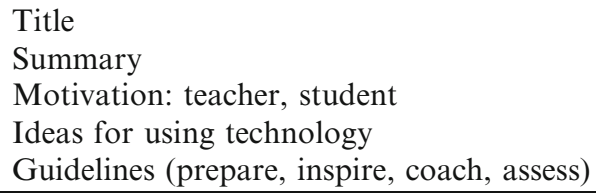

Fig. 3.7 Learning Activity template for cycle 5 and Edukata

outcomes, motivations for teachers and students to perform the activity, and reasons for using technology.

The template for a Learning Activity changed as the cycles of piloting progressed. While the main elements remained throughout the process, the wording and explanations for them did change to avoid misunderstandings. Figures 3.6 and 3.7 show the template for cycle 1 and for cycle 5 respectively. Many details related to technologies and tools were removed as they were found to be not that important. For example, instead of providing reasons for using technology, we included a section with ideas for using technology. The motivational tips were retained as they were seen as very helpful, and the actual guidelines were structured differently from the first cycle.

\section{Evaluation Results}

Evaluation results from the pilot cycles show significant changes and gains in the piloting classrooms. The results are based on teacher surveys, interviews, and diaries, as well as student surveys. A quantitative analysis and comparison of pilot activities is not possible, since no two teachers did exactly the same thing. This was a design decision made early on in the project.

Focus groups and pre-pilots during cycle 1 confirmed that indeed the Learning Activities were a functional way to communicate to teachers what we hoped they would accomplish during their pilots. The granularity of a Learning Activity seemed to be suitable for teachers so they could look at each of the activities, understand them, incorporate some of them into their upcoming course plan, and use the tips and suggestions from the Learning Activities to create a course plan that challenged them to try new methods and tools.

The Learning Activities and their implementations by the teachers enabled their students to:

- engage in active and independent learning (84\%);

- express their ideas in new ways (89\%);

- communicate with each other in new ways ( $85 \%$ );

- communicate with their teacher in new ways $(81 \%)$;

- use digital tools to support collaboration (91\%). (Lewin and McNicol 2014) 
The teachers, in turn, reported increased

- engagement in exciting new practices $(86 \%)$;

- uptake of ICT (84\%);

- enthusiasm for teaching (73\%). (Lewin and McNicol 2014)

The library of Learning Activities was considered a valuable asset, and when national policies were aligned, the approach was seen to be likely adopted and to influence future practices (Lewin and McNicol 2014). Evaluation results of iTEC are more fully discussed in Chap. 9 of this book.

\section{Packaging the Design-Based Research Method for Teachers}

After three of the five piloting cycles, it was evident that the design process that provided each pilot cycle with Learning Activities was valuable. This was underlined by the annual review, which wanted to see this design process continue after the project. Thus, the process for the final piloting cycle was changed. Instead of repeating the same process, including the piloting of designer-created learning activities, project partners decided to create toolkits for teachers to create their own scenarios and Learning Activities. The toolkit for creating scenarios was named Eduvista, and the toolkit for designing Learning Activities was named Edukata. At the end of the project, both were combined into the Future Classroom Toolkit, although Edukata remains a separate, independent design model for teachers to use.

The challenge for the design team became how to package a complex professional design research methodology into a product that teachers could use independently with good results. Here are the main features that needed to change. Some of them were seen as challenges, while others were considered opportunities that made the process easier.

- Instead of professional designers, psychologists, cognitive scientists, graphical artists, and educators, the team includes mainly educators, and possibly students and educational policy makers.

- Instead of having a thorough understanding and practical experience of the design process, participants need to able to work with as little training as possible.

- Instead of working on design full time, the work needs to be done while working as educator (student, and educational policy maker).

- Instead of scheduling design work to span 2-3 months, the work needs to be completed in a shorter timeframe.

- Instead of working on 6-10 scenarios simultaneously, only one or two need to be sufficient.

- Instead of addressing challenges of all European teachers, the scope needs to be local. 


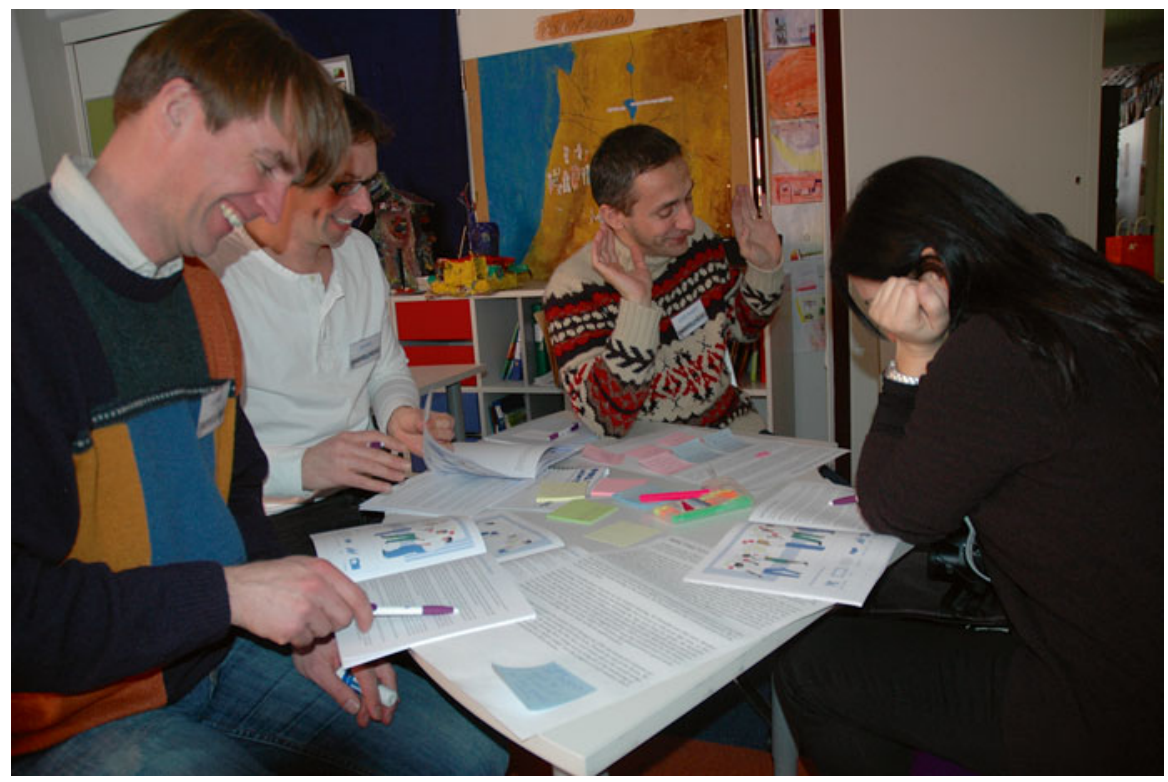

Fig. 3.8 A team of four teachers engaged in Learning Activity design in March 2013 in the iTEC Winter School. Each has the first prototype of the toolkit as a book in front of them

Much of the packaging of the design process was about simplification. Our concern was that essential parts and nuances of the process may be lost in pruning, and initially we were not sure this process would even be possible without an experienced designer facilitating the work.

We organized a 3-day workshop in the winter of 2013 in Finland, inviting 40 teachers from around Europe to attend (see Fig. 3.8). With them, we piloted the first prototype of the design toolkit. Based on observations and feedback, we continued our work, rewriting sections and simplifying them further. By the summer of 2013 a new version of the guidebook was available, and we named it Edukata.

During each cycle, national coordinators had organized workshops for their piloting teachers where the pilot materials were presented and worked through. The plan was that in cycle 5, at the end of 2013, national coordinators would train piloting teachers to facilitate the Edukata process, and each trained teacher would organize an Edukata design workshop with their colleagues. After these design workshops, teachers would use the Learning Activities they designed to plan their spring 2014 classroom pilots.

During this pilot, most national coordinators organized an Edukata design workshop with their teachers. In these design workshops, the coordinators acted as facilitators, instead of the teachers. Evaluation data showed that Learning Activities designed by the teachers themselves seemed to provide even better results than the ones in previous cycles. This was perhaps mostly due to the added freedom the teachers had, and the ability to address locally relevant challenges. 
The suitability of Edukata facilitator materials in allowing teachers to act as design facilitators, however, was not shown, as the national coordinators enacted that role. What was evident from the results, and from workshops our design team organized independently, was that the Edukata facilitator guidebook required an additional complete rewrite, to further clarify some aspects of the process, remove design jargon, and structure the process more clearly. We needed to strike a fine balance in describing the fluid structure of the process, so that the end result suggests an open process without appearing to be without structure.

Additionally, as design researchers, we were concerned that calling Edukata a 'participatory design model' was not any more warranted, as the continual simplification of the model had reduced the role of participants to that of commenting on the work in progress. So in the final iteration, with consultation from other participatory design professionals, we provided more depth to the participation aspect of the model. While we still allowed simple commenting, we encouraged teachers to involve others in more meaningful ways, as co-designers. We presented the various participation levels as a spectrum, where the facilitator may move, depending on the circumstances.

The final rewrite was finished in May of 2014, and the final, version 1.0 Edukata facilitator guidebook was published in June 2014 and translated to various European languages during the following months.

\section{Conclusion: Edukata}

All educational institutions are changing, as new technologies bring new ways of acquiring, assimilating, and adapting information. Rather than reacting at the last possible moment, all schools can proactively look into the potential futures and take steps to incorporate new possibilities and challenges into their everyday practice.

Participatory design, or co-design, is a method for crafting design ideas that may be more likely to be adopted by the people they are designed for, because of their involvement in the design process, shaping and forming the artefact and tool into use. The outstanding evaluation results of the iTEC project show that thorough participatory design situations, when teachers and students are active contributors and designers of their own working environment, may produce lasting, significant improvements in the working cultures and practices of schools.

Our research question was:

\section{What kind of support, training, materials, and experience is needed for teach- ers to create their own Learning Activities that produce beneficial results in their classrooms and those of their peers?}

Edukata, the participatory design model, is our best hypothesis for answering this question (see Fig. 3.9). Edukata is a flexible and attractive model for approaching change management by utilising participatory design practices. The model consists of an iterative sequence of design workshops, which are prepared and organized 


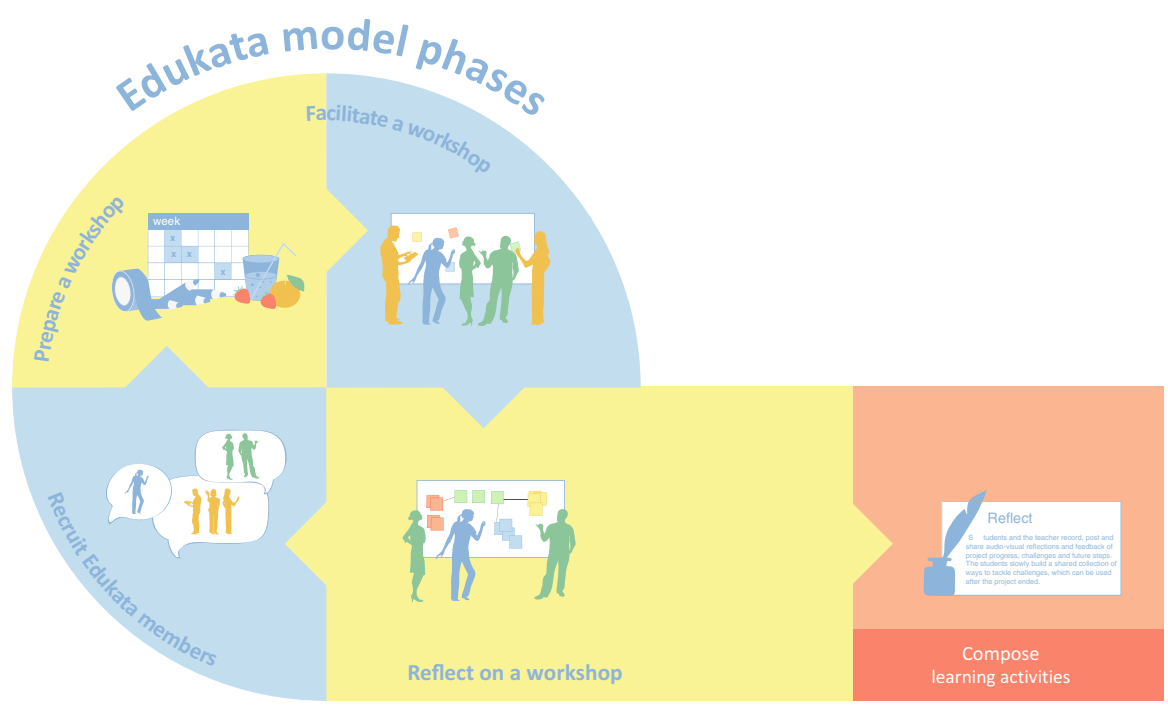

Fig. 3.9 The design process according to the Edukata model. Iterative progression of several workshops ends with writing new Learning Activities. Each workshop may involve partly different participants, so recruitment precedes other preparations. Each workshop is followed by a reflection session

by a trained facilitator. The process produces Learning Activities and learning stories, and as intermediate results, design challenges and design solutions related to the local context of the participants.

The Edukata model is described in a facilitator guidebook, which highlights aspects of each workshop phase (see Fig. 3.9), including how to recruit participants and how to engage everyone in an iterative design process. To ensure the quality of the design processes and their results, a tiered accreditation system has been set up, where people attending a facilitator workshop (see Fig. 3.10) and then facilitating a design workshop will be publicly recognized as Edukata facilitators. Several partners of the iTEC project have started their own national programmes related to teachers' continual professional development and teacher training that is including the Edukata model. Our hope is that by training more teachers to be proficient design facilitators, through these professional development opportunities, our own training workshops as well as Future Classroom training through the European Schoolnet, the contextual and adaptive aspects of Edukata will sustain without turning into a rigorous planning phase that precedes course planning.

When national policies call for renewed school practices, Edukata is a practice that can be used to turn those new policies into concrete activity ideas for teachers. When policy is defining the principles and direction, Edukata can be a way for teachers to help make them real in a school and classroom level. 


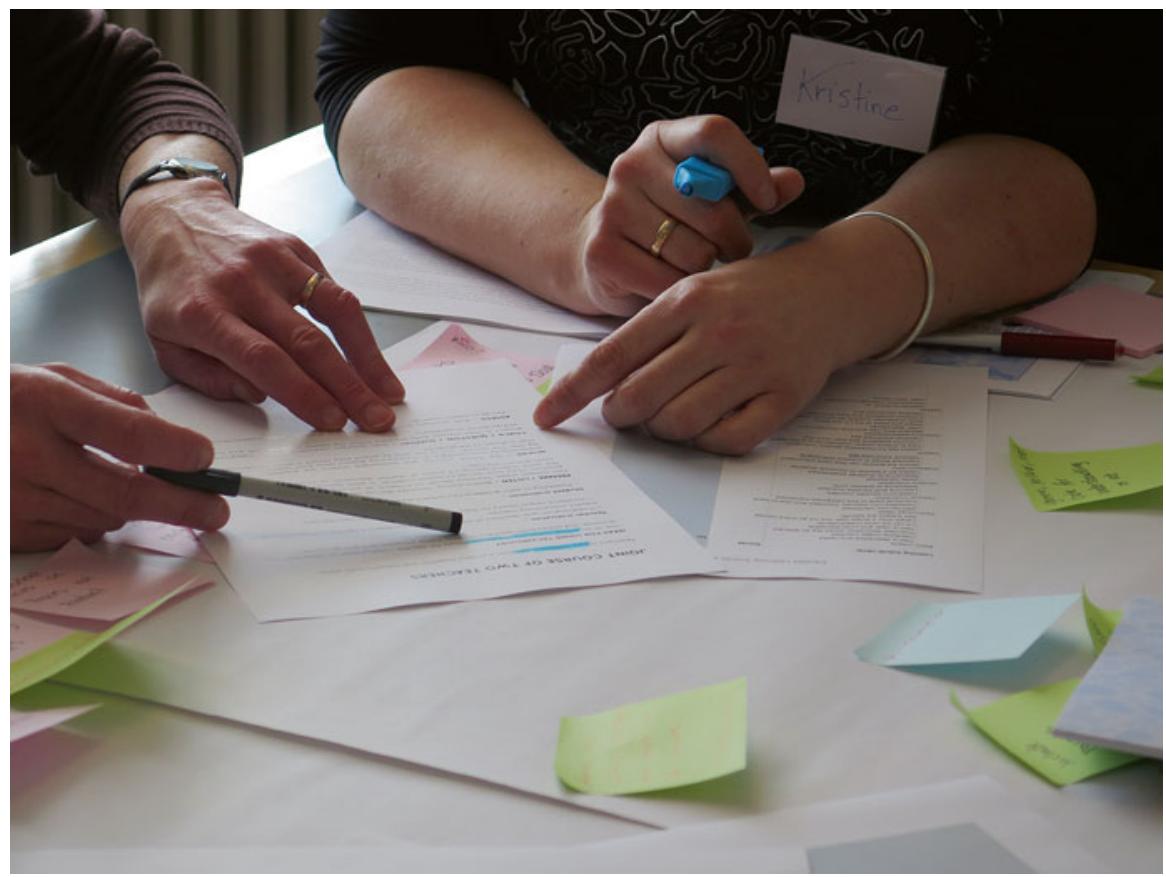

Fig. 3.10 Danish teachers working in an Edukata facilitator workshop in March 2014. The workshop consists of various exercises, including scenario analysis, simulated participatory design, challenge and solution design, and Learning Activity authoring. In this picture, teachers are evaluating an example to gain an understanding of the features of a high quality Learning Activity

As iTEC has drawn to a close, the design work can still be carried out by designers and teachers. Edukata is the design model that has been crafted specifically for educators, so they can work with their colleagues in facing new challenges and opportunities in a structured, creative, and productive manner. Edukata is part of the European Schoolnet's Future Classroom Toolkit and training programme, and also an independent participatory design model that can be used with existing scenarios. The website edukata.fi contains the guide book in several European languages, a library of existing scenarios and Learning Activities, as well as a listing of accredited Edukata facilitators and service providers.

All materials are published under an open CC BY-SA license at http://edukata.fi.

Open Access This chapter is distributed under the terms of the Creative Commons Attribution Noncommercial License, which permits any noncommercial use, distribution, and reproduction in any medium, provided the original author(s) and source are credited. 


\section{References}

Cole M (1996) Putting culture in the middle. Cultural psychology: a once and future discipline. Harvard University Press, Cambridge, pp 116-145

Conole G (2010) Learning design—making practice explicit. In: ConnectEd design conference, 28 June-2 July 2010, Sydney, Australia

Durall E, Toikkanen T (2013) Feeler: feel good and learn better: a tool for promoting reflection about learning and well-being. In: Proceedings of the 3rd workshop on awareness and reflection in technology-enhanced learning, pp 83-89

Ehn P, Kyng M (1987) The collective resource approach to systems design. In: Bjerknes G, Ehn P, Kyng M (eds) Computers and democracy: a Scandinavian challenge. Avebury, Aldershot, pp $17-57$

Engeström Y (1987) Learning by expanding. Orienta-Konsultit Oy, Helsinki

Engeström Y (1999) Activity theory and individual and social transformation. In: Engeström R, Miettinen R, Punamäki-Gitai R-L (eds) Perspectives on activity theory. Cambridge University Press, Cambridge, pp 19-38

Ford M, Leinonen T (2009) MobilED-a mobile tools and services platform for formal and informal learning. In: Ally M (ed) Mobile learning: transforming the delivery of education and training. Issues in distance education. Athabasca University Press, Edmonton, pp 195-214

Hakkarainen KAI (2003) Emergence of progressive-inquiry culture in computer-supported collaborative learning. Learn Environ Res 6(2):199-220

Keune A, Leinonen T (2013) Square1 Prototype: build your own devices for collaborative learning. In: Tuovi 11: Interaktiivinen tekniikka koulutuksessa 2013-konferenssin tutkijatapaamisen artikkelit. Tampere University

Keune A, Toikkanen T, Purma J, Leinonen T (2011) Deliverable D3.1: 1st report on design prototypes and design challenges for education. http://itec.eun.org/c/document_library/get_file?p_1_ id $=10307 \&$ folderId $=37321 \&$ name $=$ DLFE-1641.pdf

Leinonen T (2010) Designing learning tools, methodological insights. Aalto University, Espoo

Leinonen T, Kligyte G, Toikkanen T, Pietarila J, Dean P (2003) Learning with collaborative software- a guide to fle3. University of Art and Design Helsinki, Helsinki

Leinonen T, Toikkanen T, Silfvast K (2008) Software as hypothesis: research-based design methodology. In: The proceedings of participatory design conference 2008. Presented at the participatory design conference, PDC 2008. ACM, Indiana University, Bloomington

Leinonen T, Keune A, Veermans M, Toikkanen T (2014) Mobile apps for reflection in learning: a design research in K-12 education. Br J Educ Technol. doi:10.1111/bjet.12224

Lewin C, McNicol S (2014) Creating the future classroom: evidence from the iTEC project. Manchester Metropolitan University, Manchester. ISBN 978-1-910029-01-5

Muller MJ, Kuhn S (1993) Participatory design. Commun ACM 36(6):24-28. doi:10.1145/ 153571.255960

Nelson H, Stolterman E (2003) The design way: intentional change in an unpredictable world: foundations and fundamentals of design competence. Educational Technology, Englewood Cliffs

Rittel H (1972) On the planning crisis: systems analysis of the "first and second generations". Bedrifts Okonomen 8:390-396

Schön D (1987) Educating the reflective practitioner: toward a new design for teaching and learning in the professions. Jossey-Bass, San Francisco 